\title{
Widening requirements capture with soft methods: an investigation of hybrid M\&S studies in health care
}

\author{
J H Powell* and N Mustafee \\ University of Exeter Business School, Streatham Court, Rennes Drive, Exeter, Devon EX4 4ST, UK
}

A simulation study consists of several stages: problem formulation, model implementation, verification and validation, experimentation and output data analysis. The application of multiple techniques in the model implementation stage is referred to as hybrid simulation, which we distinguish in this paper from a hybrid $M \& S$ study, the latter referring to studies that apply methods and techniques from disciplines like Operations Research (OR), Systems Engineering and Computer Science to one or more stages of a simulation study. Our paper focuses on the contribution of soft OR methods in the problem formulation stage of a simulation study (and by extension a hybrid M\&S study). Soft Systems Methodology (SSM) has, arguably, been the most widely used qualitative approach for eliciting system requirements. In this paper, we present Qualitative System Dynamics (QSD), a soft systems method, as having potential use in the problem formulation stage of a healthcare M\&S study. The contribution of this paper is thus twofold: (1) a review of the literature in SSM for healthcare operations management and (2) an examination of QSD as an additional soft OR method, complementing (rather than supplanting) existing approaches, which can further aid the understanding of the system in the problem formulation/conceptual modelling stage of a hybrid M\&S study.

Journal of the Operational Research Society (2017) 68(10), 1211-1222. doi:10.1057/s41274-016-0147-6;

published online 21 December 2016

Keywords: simulation; health systems; soft systems; soft systems methodology; qualitative system dynamics

\section{Introduction}

Simulation methods enable stakeholders to analyse and evaluate strategies for effective management of complex systems, providing an experimental test bed or environment for different stakeholders to assess the effectiveness of putative, sometimes emergent policies. They can aid the investigation of both the effects of policy and problem/ system cognition (Powell and Coyle, 2005). By policy investigation, we mean the exploration of the (predicted) effects of emergent, developing policy by interested parties in the multi-stakeholder environment. Here, the scope of the system-in-focus, including its performance measures, is held constant or is at least undebated. This allows exploration of a variety of policy options, which may be operational or strategic in nature, aimed at managing the undebated system to produce optimal, desirable or at worst acceptable results. In the simplest case there is one dominant system owner who has the authority, de jure or de facto, to represent any other stakeholders in assessing system output. More often, there are a number of stakeholders vying for control over the system performance through the application of the candidate policies.

*Correspondence: J H Powell, University of Exeter Business School, Streatham Court, Rennes Drive, Exeter, Devon EX4 4ST, UK.

E-mail: j.h.powell@exeter.ac.uk
For the purposes of this work, it is taken as given that the problem they work on will require a quantitative approach and the use of computer simulation. However, this is not the same as making an assumption that simulation is the only approach, and indeed, the application of only soft methods may be found to be sufficient in certain problem contexts. Further, we recognise that many of the problem formulation difficulties specific to hybrid simulations are common to other wicked problems, but leave investigation of these parallels to later work.

In many cases, and in health systems as an example, the stakeholder set disagrees not only about the desirability or optimality of system outputs, but about the very definition of the system under scrutiny. In most cases, these disagreements are cognitive in nature since the divergence among the stakeholders' perceptions of the system is shaped by their unique experiences of working with the system. In other cases, the differences are structural in the sense that different stakeholders will define the system itself or parts of the system differently from their points of view (and in rare cases existentially), but we label them here collectively as requiring cognitive exploration in contrast with the need to explore policy options. In both cases, simulation can assist in that exploration, but the cognitive case requires, we submit, a degree of extra support.

We are concerned in this paper with healthcare studies and the application of qualitative methods for eliciting system 
requirements in the conceptual modelling stage. Soft Systems Methodology (SSM) has been the most widely used qualitative approach for this purpose, and we therefore review the literature of SSM to get a better understanding of its breadth of application in healthcare studies. The literature review is the first contribution of this paper.

This review prompted an investigation of the use of soft OR techniques complementary to SSM in the problem formulation/conceptual modelling stage. Our findings suggest that there is little evidence of this. This observation in turn provoked an investigation into the use of the well-known system dynamics technique in its qualitative form (QSD) as an additional soft OR method, complementing existing approaches, in the problem formulation/conceptual modelling stage of a healthcare study. This is our second contribution.

The remainder of the paper is organised as follows. Section 2 presents an overview of hybrid M\&S studies and a review of techniques from other disciplines in the conceptual modelling phase. Section 3 is on soft OR, its applicability in multi-stakeholder environments, description of some of the methods and historical context. The literature review of Sections 4 and 5 identified the lack of use of complementary soft OR techniques (which could potentially be used together with SSM) in the problem formulation stage of a hybrid M\&S study. In Section 6, we critique this segmented approach and argue the need for soft OR techniques that not only provide insights into the 'wicked or 'messy' problems but further support the development of the quantitative simulation models and could potentially be used together with SSM. We discuss, specifically, the use of qualitative system dynamics (QSD) (Powell and Coyle, 2005), a modelling and structural analysis technique which bridges the numerical, positivist requirements of simulation and the socially constructed world of its surrounding context. It replaces the detailed simulation-type system output with a structural analysis, concentrating on predicting desirable managerial interventions by direct consideration of the dominant dynamics of the system-in-focus. As such we find its ability to incorporate system knowledge other than that of a numerical nature highly conducive to the requirements capture of simulations in the present context. The concluding section of our paper presents future work.

\section{Hybrid M\&S study}

Health systems have particular characteristics in terms of requirements capture, including an unavoidable dissonance between the ontologies of the simulated system (positivist, single reality) and that of the surrounding socially constructed, multiply-defined recipient and policy contexts. Within healthcare studies, simulation techniques such as Monte Carlo simulation (MCS), discrete event simulation (DES), system dynamics (SD) and agent-based simulation (ABS) are in wide use (Brailsford et al, 2009a, b; Katsaliaki and Mustafee, 2011). Frequently, however, these techniques have been applied in isolation one from another, in dissonance with good system practice (Jackson, 2000), which stresses the usefulness of hybrid methods in overcoming the limitations of any single approach. The search for the best possible representation and analysis of the system under scrutiny has, then, led to an increase in the use of combined multiple simulation techniques in the model implementation stage (Chahal and Eldabi, 2010; Viana et al, 2014; Fakhimi et al, 2015), thereby enabling synergies across techniques. While this implementation stage is critically important, the overarching modelling and simulation (M\&S) study has other well-defined stages (Maria, 1997), for example, conceptual modelling (Robinson, 2011), data collection, validation and verification, model execution, output data analysis and recommendations. It is, then, appropriate to explore the use of methods and techniques from disciplines like Operations Research (OR), Systems Engineering, and Computer Science to one or more stages of a simulation study. The focus of this paper is the conceptual modelling, which is a vital stage of model development but is the least understood aspect of a simulation study (Law, 1991).

The existing literature in hybrid $\mathrm{M} \& \mathrm{~S}$ studies mainly comprise of discursive papers comparing different techniques (e.g. Brailsford, Desai and Viana, 2010), case studies, integration frameworks and studies that have used the frameworks/framework extensions to create hybrid models (e.g. Chahal and Eldabi, 2008, 2010; Zulkepli et al, 2012; Viana et al, 2014; Fakhimi et al, 2015; Mustafee et al, 2015). Specific techniques that have been applied include: the combined application of SD and DES (Viana et al, 2014; Zulkepli et al, 2012; Chahal, 2010; Chahal and Eldabi, 2008), models using ABS, SD and DES (Djanatliev and German, 2013; Viana et al, 2012), combined application of ABS and load plan construction heuristics (Mustafee et al, 2012), loosely coupled SD and agent-based models (Djanatliev et al, 2012) and application of integer programming with simulation (Lee et al, 2012). The majority of these papers focus on the model implementation phase of a simulation study.

Our review of the literature provides evidence of the application of methods and techniques from disciplines like Computer Science, Systems Engineering, OR, Information Systems and Distributed Computing to the various other stages of an M\&S study. For example, in problem formulation stage approaches from soft OR, systems engineering and information systems have been used (refer to the previous section for specific examples). For faster simulation execution in the experimentation stage, authors have used techniques from computer science, e.g. general purpose computing on graphics processing units (GPGPUs) (Perumalla, 2006; Park and Fishwick, 2010), parallel and distributed simulation (Lendermann et al, 2001; Mustafee et al, 2009) and distributed computing solutions like simulation execution over desktop grids (Mustafee and Taylor, 2009; Taylor et al, 2011) and, in the future, cloud computing resources. Model formalisms 
based on discrete event system specification (DEVS) (e.g. dynamic structure discrete event system specification (DSDEVS)-Barros, 1995) and meta-modelling using UML (Traoré, 2003) have been used. Further, it is arguable that statistical and computing approaches presently being developed in the field of business intelligence, big data and analytics (Demirkan and Delen, 2013) could be applied in $M \& S$ study stages pertaining to the collection of input data and output data analysis.

The discussion above indicates the multi-disciplinary nature of an M\&S study. Unlike a hybrid simulation that focuses on individual $\mathrm{M} \& \mathrm{~S}$ techniques in the model implementation stage, a hybrid M\&S study recognises and deploys the use of interdisciplinary methods at various other stages of a simulation study. Figure 1 shows our conceptualisation of a hybrid M\&S study, identifying a number of interdisciplinary methods that have been used (or can potentially be used) in specific stages of an M\&S study. Our conceptual representation is not exhaustive (indeed, not all stages of a simulation study are depicted; stages pertaining to input data and output data analysis have been combined; model formalism has been introduced as a stage). Figure 1 includes the model development stage at the centre and depicts four simulation techniques (in grey background) that are frequently used in isolation or can be combined to implement a hybrid simulation. These specific $M \& S$ techniques are distinguished from those originating from the other disciplines (shown in white boxes, e.g. UML, GPGPU execution, big data and analytics) that are/can be applied to stages such as Model Conceptualisation, Model Formalism, Input Data Analysis, Output Data Analysis and Simulation Experimentation.

Our paper focuses on the starting point of a $M \& S$ study (and by extension a hybrid M\&S study) with the objective of identifying commonly used interdisciplinary methods in the conceptual modelling stage. Through a review of the literature, we were able to identify the following: (a) systems engineering approaches and tools [e.g. SysML: systems modelling language-Eldabi et al (2010)], (b) problem structuring methods (PSM) [e.g. Soft Systems Methodology-Lehaney and Paul (1994, 1996), Kotiadis et al (2014); Group Model BuildingBérard (2010)] and (c) concepts from information systems [e.g. issue maps/issue-based information system (IBIS) Eldabi et al (2010)]. Our review of the literature also revealed the prominence of the soft systems methodology (SSM) (Checkland and Scholes, 1990) in healthcare studies in general, especially those that involved multiple stakeholders in the decision-making stage. We, therefore, considered it pertinent to conduct a literature review of SSM in health care in general. Thus, for the literature review, we were not restricted to the application of SSM in hybrid M\&S studies, but considered papers that also used a single approach or indeed other OR techniques (please refer to the keyword search strategy in the section on methodology). However, a large proportion of the papers reported on the use of SSM in hybrid M\&S studies. This is not surprising for the following two reasons;

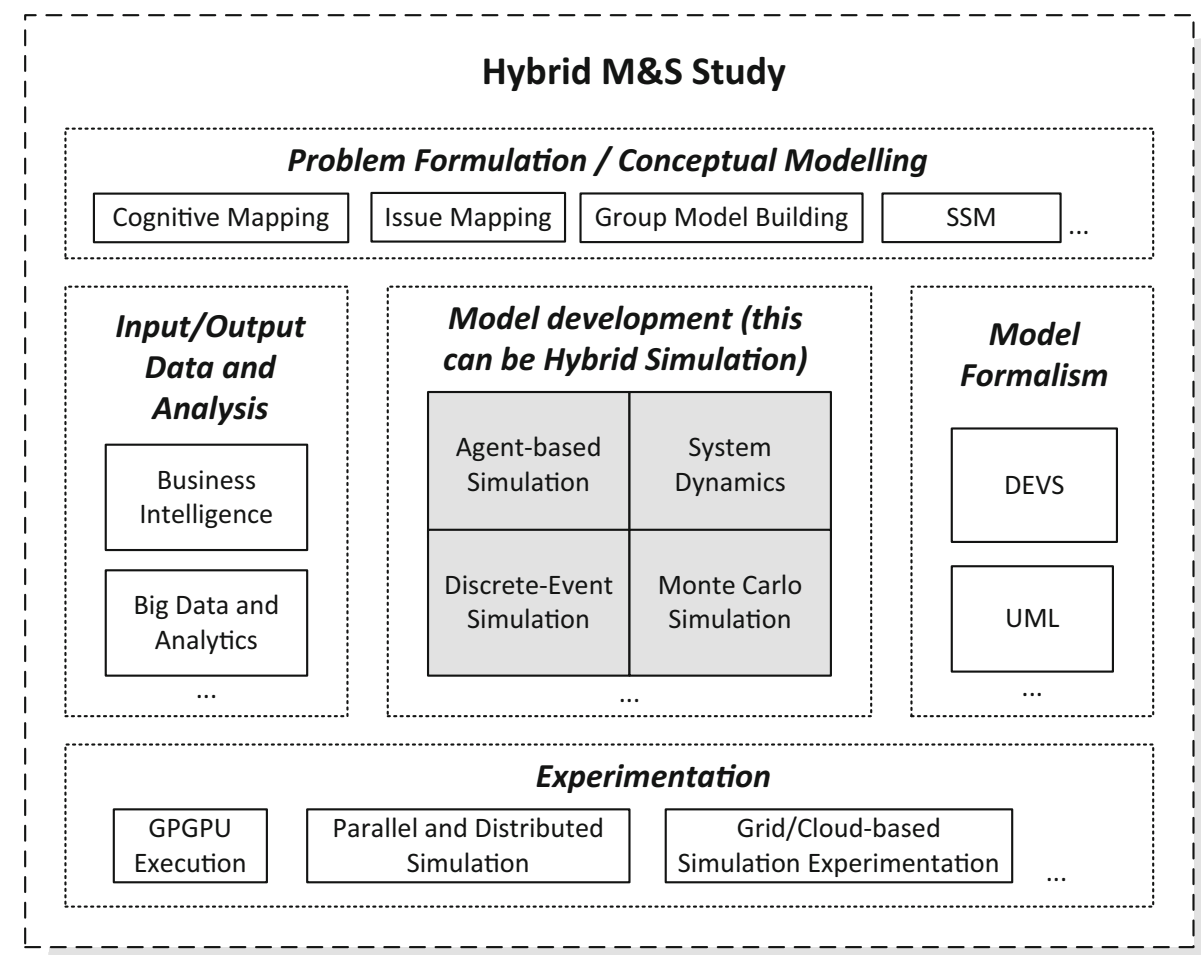

Figure 1 Conceptual representation of a hybrid M\&S study (... denotes other methods). 
(a) Simulation is a widely used OR/MS technique that is reported in the literature. Further, discrete event simulation is arguably the most frequently used classical OR technique that is applied across a range of industries including health care (Hollocks, 2006).

(b) The 2004 review of OR research in the UK reported that, 'from an international perspective, unique selling points of significant strength within the British OR research agenda are so-called "soft OR" and applications in health care' (EPSRC/ESRC, 2004).

\section{Soft OR, its applicability in multi-stakeholder environments and historical context}

Traditional OR was predominantly quantitative, using primarily what are known as 'hard' methods today. From the 1970s onwards and increasingly since, a concern with the abstraction and impracticality of much OR emerged, recognising the limitations of hard approaches. One of the major responses at the time, especially in the UK, was the development and approval of a variety of non-mathematical methodologies. These became known as 'Soft' OR or problem structuring methods and were qualitative in nature. These approaches (alone and in complementarity with hard approaches) have been particularly effective in extending the spectrum of problems to which OR can be applied. An analysis of the theoretical and philosophical underpinnings of these two fundamentally different approaches allows insights into their purposes and areas appropriate for application.

Hard OR is based on the assumption that systems are 'objective' aspects of reality-they are generally independent of the observer. As such hard OR assumes that system models are models of the world (ontologies) and that the world is composed of a system that can be 'engineered'- that can be quantitatively and objectively represented. It is believed that different observers perceive a system in the same way and that goals or objectives identified are identical. As a result, it is argued that the most substantial elements of a system can be quantified, that reliable data can be collected and that such information can usually be well represented by a numerical or computer-based model for solution development. Furthermore, the developed models do not need to be transparent to client(s), the role of the OR person is of expert nature, and future uncertainties can be modelled using probability theory.

Soft OR methods by contrast can best be characterised as assuming that a system is defined subjectively - it is a reflection of the observer's worldview. It is for this reason that system models are essentially treated as intellectual constructs (epistemologies). An individual will interpret the world based on his social, cultural and educational background and as such not necessarily offer a description of what exists in reality, but what is perceived a useful representation of interrelationships (Daellenbach, n.d.). Thus, soft OR expects there to be a range of decision-makers or stakeholders involved in defining the nature of a situation that may all have differing and even conflicting objectives. It therefore provides a strategy to accommodate for these complexities. As a result, transparency and accessibility of the model to the various stakeholders is significant, predominantly ruling out complex mathematical models. Finally, the OR person's role is of facilitative nature by guiding and structuring the various stakeholders discussions and uncertainties are not merely reduced to probabilities.

Based on these classifications, hard OR has been considered suitable in instances where the problem situation involves considerable technical complexity; however, hard OR has been found to be limited in its ability to cope with systems encompassing high human complexity and divergence of interests (Daellenbach, n.d.) — the so-called wicked problems which refer to 'social systems problems that tend to be ill-formulated, where the information is confusing, where there are many clients and decision-makers with conflicting values, and where the ramifications in the whole system are thoroughly confusing' (Churchman, 1967—quoting Prof. Horst Rittel).

A key aim of soft OR methods is to build a shared understanding of a wicked problem, which is then to be used as a starting point for discussion towards finding an appropriate resolution approach that all participants are prepared to implement. UK has been at the forefront of research in soft methods; this was highlighted in the 2014 EPSRC/ESRC 'Review of Research Status of OR in the UK' (EPSRC/ESRC, 2004) which reported on its significant strength in soft OR. We now present a summary of soft OR methods.

\subsection{Soft systems methodology}

SSM is designed to deal with complex situations where there are divergent views about the definition of a problem. It is primarily concerned with using system concepts to foster a process of learning and understanding about a given problem situation, among various individuals, to improve the situation for the parties involved by identifying areas for change. SSM is usually carried out using a seven-step process. These steps involve learning about the problem situation by also looking at social, political and human aspects in general, creating models of decisive activity, examining the situation using the models to be able to reach a consensus about the actions needed and finally taking action based on the consensus reached. This is repeated in a cyclic fashion to continuously improve a system by learning about it.

\subsection{Strategic option development analysis (SODA)}

SODA is typically employed when working with a group of people whose perceptions of a problem situation are difficult to define and coordinate. It utilises cognitive mapping as a modelling device to help record and extract the viewpoints of the various individuals involved, in order to finally produce a 
strategic map, which incorporates and merges the individual cognitive maps that were constructed. The strategic map is utilised as a framework for group discussions, which are led by a facilitator who guides participants towards committing to a range of actions.

\subsection{Strategic choice approach (SCA)}

The SCA is centred on dealing with the uncertainty usually entailed by problematic situations and decisions. Its purpose is to aid a group of decision-makers in deciding which strategy to pursue through structuring and discussing a problematic situation and possible solutions at workshops. In a SCA, the planning process is separated into four modes: shaping, designing, comparing and finally choosing. Shaping involves deciding on the problem focus as well as the areas that require decision-making. Designing comprises an analysis of the decision areas identified as most urgent, to determine available decision options and their interconnectedness/compatibility. This is followed by Comparing, where requirement criteria of a feasible and suitable strategic approach are discussed and specified. Finally, Choosing involves deciding on the options that look most promising especially when assessed against various uncertainties.

\subsection{Drama theory and confrontational analysis (DT\&CA)}

DT\&CA is a socially interactive approach that was designed specifically to address problems involving multiple parties with conflicting objectives (Stubbs et al, n.d.). It is principally constructed to explore real situations by creating an interaction in which rational choice, emotion and argument of the various stakeholders surface, to arrive at a reasonable resolution. A confrontation consists of a number of characters, which are the stakeholders involved in a problem situation. Each of these holds a position that it is promoting as the desirable solution to the other stakeholders. Characters also hold fallback positions, which are strategies that they encourage if things have not gone their way. To capture a confrontation, a representation scheme called a card table was developed. Each character has a set of cards or options that it can choose to pursue, which are mapped on a card table. Each confrontation involves characters facing drama theoretic dilemmas, which evolve through the various characters attempting to resolve them and may encourage the formation of new alternative strategies. The resolution of a confrontation is achieved via dilemma elimination.

\subsection{Qualitative systems dynamics (QSD)}

One of the most widely used systems approaches is that of system dynamics [SD], which formalises the system representation initially in the form of an influence diagram or ID (sometimes called a causal loop diagram) which captures causal linkages within the system-in-focus (Eden, 1989; Sterman, 2000). The SD literature stresses a relatively formal procedure, which can be summarised as representing the system-in-focus in a structural model, formalising that as a mathematical model, establishing a reference mode (by which is meant the baseline dynamic model of the system-in-focus) and extrapolating the behaviour of that reference mode by 'tweaking' the parameters of the reference mode to represent alternative policies for consideration.

This ID then forms the basis for a mathematical expression of the system structure which in turn is the basis for a simulation, the exercise of which, in the numerical form of analysis, achieves the time domain prediction of behaviour which is compared with that of the reference mode.

The analyst using the qualitative approach of SD (Powell and Coyle, 2005) does not have at his or her disposal the predictive capacity of a numerical model and so, rather than concentrating on dynamic behaviour, identifies the significant mechanisms (equivalent to the dominant loops of the quantitative method above) operating within the system-in-focus. This is almost always done by subjective, experientially based selection by informants.

By examining potential behaviours of these significant mechanisms, the analyst can forecast the effect of policy action on the inherent causal mechanisms. Essentially, actions are identified which will push the behaviour of the loops in the directions desired, avoiding resonant system behaviours which are undesired.

\section{Methodology}

In order to identify papers for our methodological review of the literature, we conducted a search using the ISI Web of Science $^{\text {TM }}$ (WOS) database. WOS is one of the largest databases of quality academic journals and provides access to bibliographic information pertaining to research articles published from 1900 onwards (for Science Citation Index Expanded). It indexes approximately 9800 high-impact research journals spread across more than 200 disciplines. Further, WOS now includes two conference proceedings citation databases, for Science (CPCI-S) and Social Science \& Humanities (CPCI-SSH), respectively, which indexes the most significant academic conferences (from 1990 onwards) in a wide range of disciplines. We used various combinations of keywords and varied parameters like specific WOS databases used and time span for the search, reviewed the retrieved results and subsequently decided on the specific search parameters for the study. We used the keywords ('soft system* method*') AND (health*), with * being the wildcard character which allowed us to use the keyword derivatives in the search condition, to conduct a topic search on title, abstract, author keywords and keyword plus ${ }^{\circledR}$. All WOS databases were included in the search. We did not restrict the year of publication and selected time span as 'all years'. It is to be 
noted that we did not use the acronym SSM since our preliminary searches using this keyword resulted in a number of irrelevant hits. We subsequently tried to apply a search condition which would restrict results to SSM in the context of health care; however, this did not produce the desired outcome since several papers on superficial spreading melanoma (which is the most common form of skin cancer) were retrieved.

Our search criteria retrieved a total of 68 papers for the initial analysis. The next stage involved reading the abstracts and identifying papers that applied SSM in the context of healthcare operations management. Thus, we excluded, for example, four papers that used SSM for designing healthcare information systems (including papers on eliciting user requirements for such systems) and further two papers on using SSM for planning and management of information security in health care, one paper on racial equality in health provisions, one paper on SSM-based evaluation of electronic library for healthcare professionals and one paper on using SSM to identify impact and barriers to information access and use in health. Forty-nine papers were selected subsequent to the abstract reading phase of the study, and this comprises the corpus of scholarly articles for the literature review part of this paper.

\section{Review of the literature in SSM}

In this section, we present the findings from our methodological review of the literature based on a keyword search of soft systems and health systems. The variables captured in analysing the articles included the aim of the study, the specific application context, the country in which the SSM study was conducted, whether SSM was used as per the original methodology (e.g. the seven-stage SSM process; (Checkland, 1981)) or extensions/ improvements to the methodology were proposed, software used (e.g. for the purposes of data analysis, illustration of "rich pictures'), the SSM concepts and tools that were used (e.g. holons, 'rich pictures', root definition, CATWOE, conceptual model, etc.), the primary stakeholders, the composition of the stakeholder group who contributed to the development of the conceptual model(s) and/or who were responsible for recommending and/or implementing the results through 'action research', outcome of the study/findings, how SSM contributed to the outcome of the study, and whether the SSM study included elements of mixed methodology (considering the scope of this paper, this variable is of particular interest to us and will be further explored). Further to this, for studies which had used SSM in the context of M\&S, we collected variables describing the type of simulation model (e.g. DES, SD) and whether the paper included the implementation aspects of the aforementioned model.

Approximately $80 \%$ of the 49 papers selected for review (40 papers) have been published in journals (one exception to this is a poster which was published in the journal Tropical Medicine \& International Health); the remaining nine papers were published as conference proceedings with Winter Simulation Conference accounting for six papers. In relation to journals, 11 articles have been published in the Journal of the Operational Research Society, followed by three articles each in the Journal of Advanced Nursing and Health Services Management Research (refer to Table 1).

It is to be noted here that our dataset included review papers (paper number 10 and 38-see Table 1), papers which proposed methodological extensions to $\operatorname{SSM}(17,48)$ and frameworks (28), discussion and viewpoint papers $(27,41)$ and empirical papers reporting on SSM studies. With regard to the latter, most of the studies were based in the UK (20 studies); this is hardly surprising since the founder of SSM (Peter Checkland) and his colleagues were based at Lancaster University (UK) and spent decades working on approaches that finally culminated in the formalisation of SSM as a problem structuring approach. Further, it is widely recognised in the international OR/M\&S community that Britain has significant strengths in 'soft OR' (and applications in health care) (EPSRC/ESRC, 2004). Studies that were conducted outside the UK include three studies in the USA (in Chicago-31, multiple regions-38, 35), one study each in Australia (State of Victoria) (18), South Africa (KwaZula-Natal) (19), Ireland (23), Norway (33), Brazil (36), Greece (37), Iran (39) and Canada (41).

A few studies have proposed an extension to SSM. The study by (12) introduces SSM + that consists of 16 stages and is positioned for the use of policymakers, clinicians and managers in healthcare circles. The study by (32) proposes the extension of SSM's graphical elicitation with graphical modelling approaches from other disciplines. The study by (40) proposes the inclusion of performance measurement model (PMM) in SSM. However, the majority of the studies either have 'religiously' adhered to the seven-stage process of SSM (e.g. 25,20) or have adopted a subset of SSM concepts and tools (e.g. there are references to using 'SSM as a guiding principle', using 'SSM-inspired tools', using 'modified version of the methods from SSM'). Our dataset also consisted of at least eight papers that claimed to have conducted a SSM study; however, the process itself was not adequately described/the use of SSM was not apparent, and in some cases, only a general reference to SSM was made. It is arguable that a couple of these studies used the term SSM as an umbrella term since their case studies included multiple stakeholders with a need to form consensus/cooperatively implement actions. We had two papers in which a plan for action was proposed, i.e. the SSM study was not yet implemented and the authors articulated the future course of action $(44,35)$.

Our next analysis is the context of the application or the specific theme of study. Some of the themes we identified included use of SSM for professional and organisational learning-including development of curriculum in health sciences $(15,21,33)$, evaluation of decision-making in interagency/intra-agency, interdisciplinary/unidisciplinary teams $(16,34)$, use of SSM for improving patient care (18, 23,30 ), critical systems inquiry into the complexities of implementing healthcare provision $(19,20,25)$, use of SSM for conceptual modelling (32), the use of SSM for multiagency planning of disaster (12), etc. 


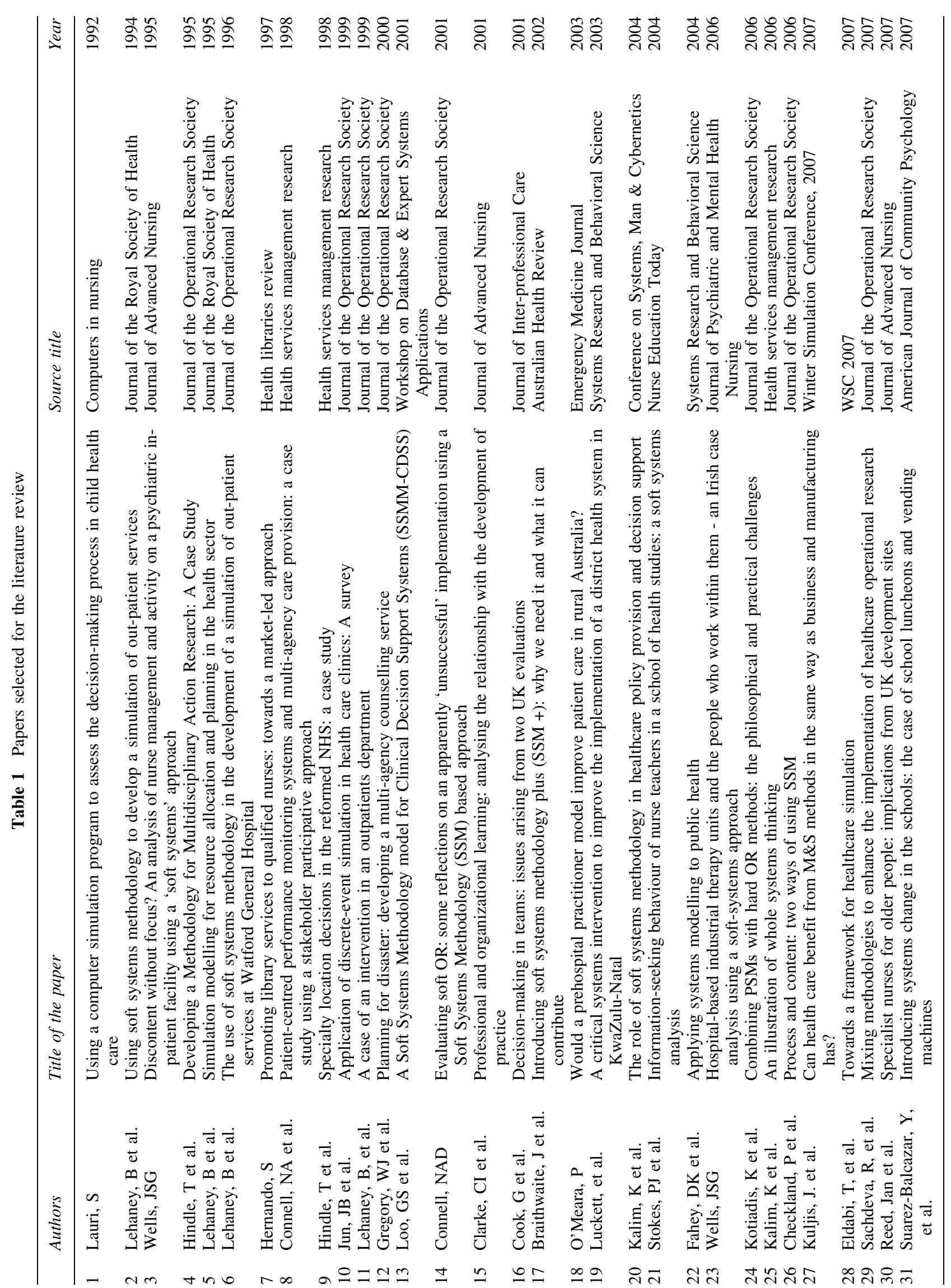




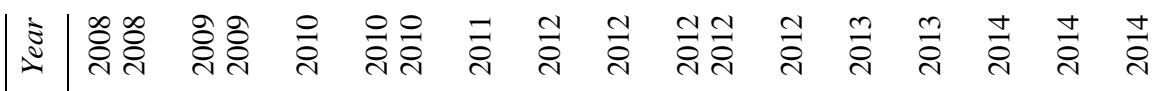

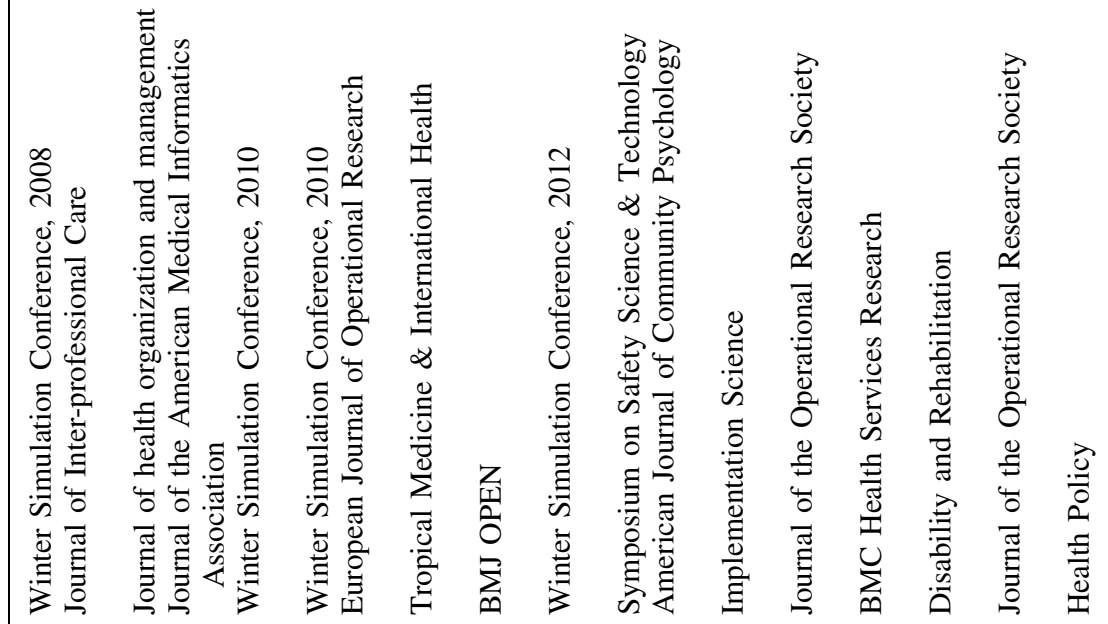

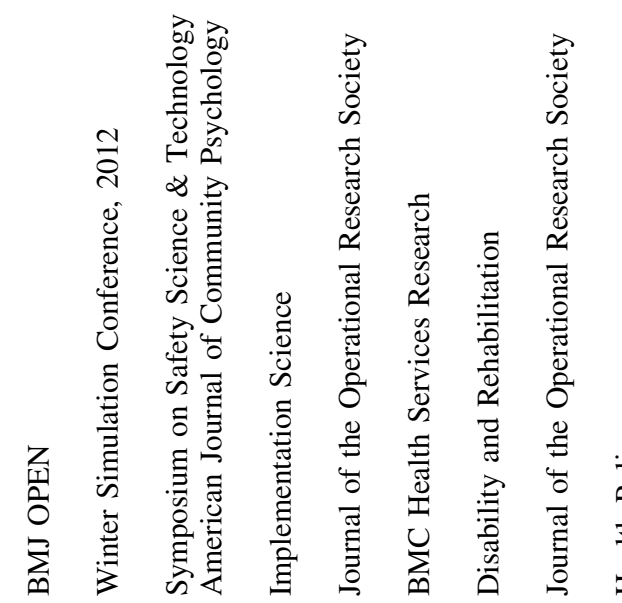

吾

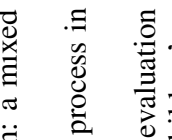

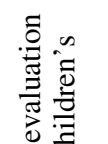

है है

$\cong$

气ั๊

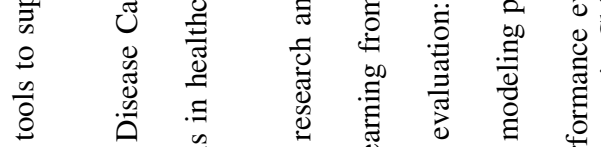

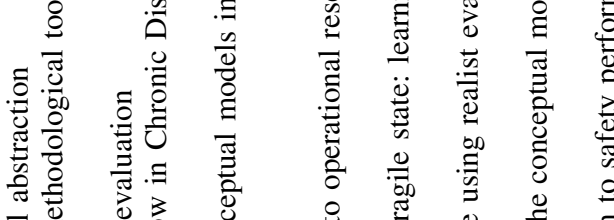

ฮ

告

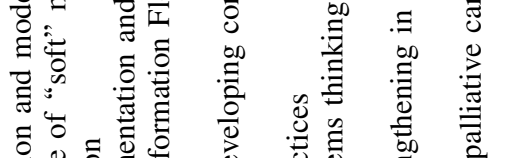

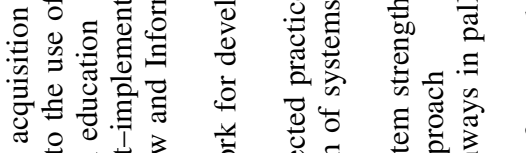

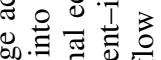

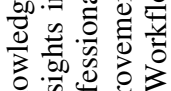

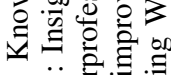

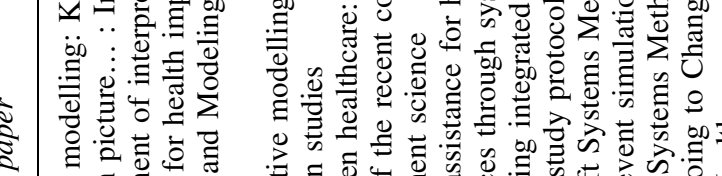

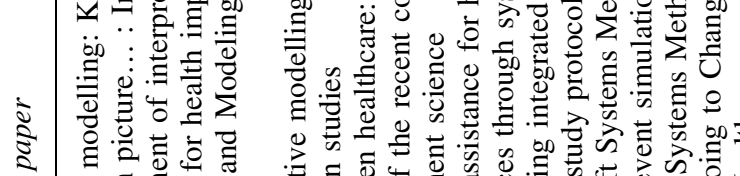

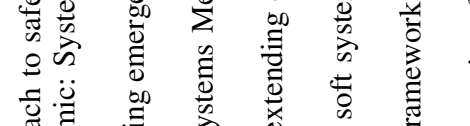

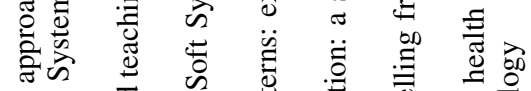

品

일

章

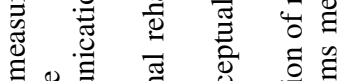

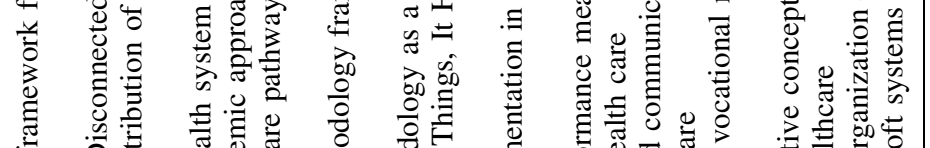

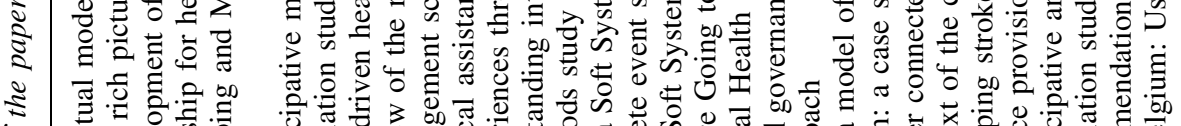

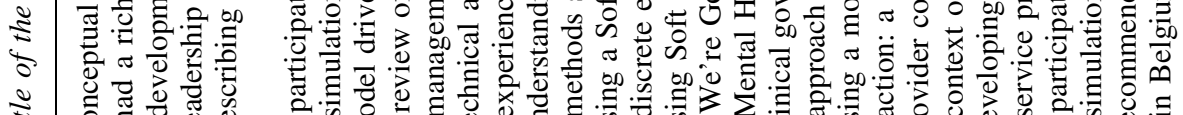

齐

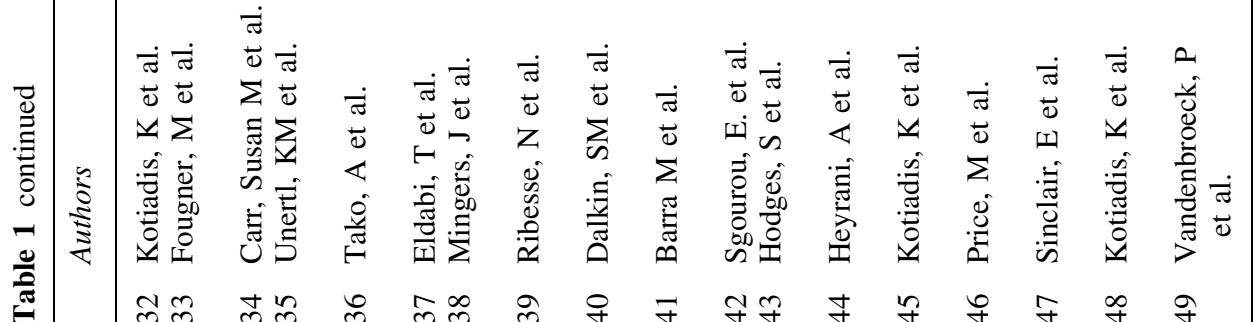


Our final analysis focuses on the complementary methods that have been used together with SSM in the development of conceptual models (it is to be noted that the development of the conceptual model is the fourth step in the seven-stage SSM process; for an M\&S study that uses SSM, the SSM conceptual model informs the conceptual model of the simulation to be developed). Of the 49 studies in our literature review, we could find evidence of multiple methods in only a handful of studies. The study by (16) advocated two approaches for evaluating the success of integrated, multi-professional healthcare teams in the UK-SSM and a pluralistic framework; however, these approaches were not used together but in two separate case studies. Thus, it is arguable that this particular study has not implemented a mixed methodology. The study by (19), however, contributes to the growing literature on the combination of systems methods by illustrating how conceptual models of SSM's purposeful human activity were constructed from the participatory use of Concept Maps and Sign-graph Diagrams. The study by (26) has used SSM with a project management framework (PRINCE2); this is an example of using a concept from operations management (project management) together with soft OR. The study by (31) has used a social ecological model together with SSM, wherein the former was used to understand the interaction between various personal and environmental factors, and the latter helped 'understand the multiple perspectives of stakeholders which in this case were also a source of resistance to change and conflict'. In (34), an appreciative inquiry was combined with SSM to form an evaluation approach that was designed to capture individual as well as organisational learning. In (35), the authors developed models of workflow and information flow using Hierarchical Task Analysis and SSM. Thus, only a handful of studies have explored the use of complementary approaches that can be used together with SSM, and this, we believe, is an area of future research.

\section{Complementarity requirements}

It is clear from our review of the literature that there is little evidence of the mobilisation of complementary soft OR techniques in the problem formulation/conceptual modelling stage of the hybrid M\&S approach. This is somewhat surprising in light of the clear applicability of SSM, in particular, to computer science applications and IS specification (Checkland and Scholes, 1990: p53) and its claims to relevance to harder approaches (for example, the inclusion of 'other systems thinking' in the original 7-stage process of SSM (Checkland, 1981; Kotiadis, 2007; Robinson, 2008)). Regardless of the reasons why that complementarity does not appear in M\&S practice, there exist dangers in such a segmented approach, particularly in respect of the need for the inclusion of:

(a) the maximum information about the behavioural dynamics of the system under consideration. The very raison d'etre of a simulation (at least in this context) is accuracy of dynamic representation, in order to extrapolate the effects of system mechanics in pursuit of comparative assessment of policies. Knowledge of these dynamics is held by various stakeholder groups, demanding the multiple perspective which is the strength of soft OR methods. If this is elicited in an explicit manner, there is an additional advantage of being able to validate, together with the stakeholder group, the functional behaviour of the simulation on which the whole policy assessment turns.

(b) the value perspectives of stakeholders other than the system owner. At first glance, it may seem that the multiple valuations of system outputs can be respected ex post facto, by giving voice and weight to the system outputs in the weighing of the benefits of the proposed policy or policies. To some extent, this can be done, but there is a danger that in declaring and designing the output representations within the simulation, those implicit valuations are made inadvertently, rendering less visible, or even invisible, factors valued by a stakeholder group which happens to be under-represented in the requirements capture process. To decode, modellers run the risk of ignoring the legitimate valuations of interested but silent parties.

(c) differing definitions of the system-in-focus. Conventional requirements that capture processes and techniques are focused on the establishment of a single simulation definition, not least because this makes subsequent model building easier. While clarity in the simulation definition is much to be valued, work in a variety of embodiments of the soft system philosophy (Checkland, Jackson, Oliga, Coyle...) shows that the single clarified reality of a model representation is often, in fact, a misrepresentation, an over-simplification of a multiple reality produced by the varying, equally valid cognitions and ontologies of stakeholders. Again, to decode, modellers run the risk of assuming that while various stakeholders may have different valuations of system outputs, they, nevertheless, agree on the definition of the system each is trying to optimise. This is a risky assumption; the various valuations of stakeholders often lead them into differing cognitions of the system and therefore of its definition. At best, the single simulation definition is the result of the interplay of power and communicative capacities of the stakeholders. Now, the nature of the simulation component of the overall assessment system is such that a single requirement (definition of the simulation) is needed at any one time, so that the multiple stakeholder definitions of the system must be dealt with accordingly. There are two options: one is to reconcile differences in system definition among the stakeholders and the other is to run variations of the simulations to reflect the varying 'flavours' of system perceived by the stakeholders. In respect of the reconciliation approach, while this is much 
to be favoured in terms of the necessary resources applied to simulation (only one simulation is needed), we note that the resolution of stakeholders' views may be different during the requirements capture phase from the policy assessment phase. This adds further weight to the need for an overall assessment process which allows for policy consequences to be included in the early stages of simulation requirements capture.

These perspectives on the plural, even arbitrary nature, of the social and political contexts surrounding a human activity system (HAS) (Checkland, 1981), of which health systems are clear examples, are, of course, widely offered. There is a clear ontological disparity between the hard, positivist, physical reality of, for example, of the physicality of a supply chain and the socially constructed, politicised, multi-perspective view of the degree of benefit provided by a health delivery system. In fact, the whole issue of the reconciliation of these ontologies can be argued to be the concern of the whole corpus of work known as soft OR (Ulrich, 1988; Checkland, 1981; Mingers and Lyons, 2009). The clash of ontologies, then, is well known, and therefore, what is worthy of note here is that there is so little practical mobilisation of this corpus of soft OR methods directly into the world of simulation, particularly at the requirements capture stage, particularly when the influence of SSM and strategic options development analysis (SODA) (Eden and Ackermann, 2004) being effective broad cognitive and problem-solving tools, is so great within health system studies.

The explanation, we assert, lies in the level at which systems-in-focus are treated by the soft OR methods. The strength of these approaches lie in their capacity to treat the broad problem in a broad manner. It is not their objective to make precise the mechanics of the underlying system which is the legitimate and complementary focus of the simulation. We hasten to add that this shortcoming, seen from the point of view of the simulation worker, should not be seen as a criticism of the soft OR arsenal, any more than a field gunner can be criticised for not being a sniper, but, from the point of view of achieving helpful, applicable, representative requirements on which basis simulations can be constructed, an interlocution between the powerful but broad, multivalent and plural structures of soft OR and the precise, but narrowly defined structures of simulation would appear highly desirable.

The three issues detailed above provide some basis for declaring the characteristics of a complementary approach through which simulation requirements analysis, particularly in the health studies area, can be improved. It needs to be able to

(a) Represent and capture expert knowledge of expected system dynamics

(b) Represent and capture available expert knowledge of the system structures underlying those dynamics

(c) Allow the coexistence of alternative/conflicting evaluations of system behaviour (d) Allow the coexistence of alternative/conflicting system structures

While a number of the well-known techniques of soft OR, including SSM, SODA (Eden, 1989), drama theory, scenario planning, general causal mapping, theory of constraints and benefits modelling (Vidal, 2005) provide very powerful insight into the nature and characteristics of complex problems (often referred to as 'wicked or 'messy' problems), they invariably do so not by detailed modelling of the dynamics of a system (as in simulation and quantitative systems dynamics) but by more discursive, verbal, often diagrammatic representations. Two stand out from the field in respect of their ability to support directly the simulation activities. These are SODA's cognitive mapping and QSD, the qualitative form of SD, and particularly the variation known as qualitative politicised influence diagrams (QPID) (Liddell and Powell, 2004; Swart and Powell, 2006; Powell and Swart, 2010). We do not reject SODA as a basis for requirement capture here, but offer the observation that because of QSD's provenance as emerging from the corpus of quantitative $\mathrm{SD}$, it has a natural structural connection with the vocabulary and representation methods used by SD to scope and specify its form of simulation and hence provides a natural interface with the needs of simulation in the health systems area in particular.

\section{Conclusion and future work}

A hybrid M\&S study is characterised by the use of methods from fields such as OR, Computer Science, Systems Engineering, Information Systems and Distributed Computing; these methods are applied to specific stages of a simulation study. Unlike hybrid simulation that focuses on the combined application of multiple M\&S techniques in the model implementation stage (e.g. system dynamics with agentbased simulation), a hybrid $M \& S$ study recognises the use of interdisciplinary methods in the wider simulation study. The focus of this paper is on the conceptual modelling stage of a healthcare M\&S study and the application of soft OR methods.

Conceptual modelling is a vital stage of model development, but is the least understood aspect of a simulation study (Law, 1991). It has been identified as potentially the most significant stage in a simulation study because the design of the model and its ability to validly reflect the real system will affect all aspects of a study. A possible explanation for the lack of attention to this aspect of modelling can be traced back to the fact that abstracting a model from a system, as done by conceptual modelling, is more an art than a science, making a universal description of methods and techniques complicated. If the conceptual model developed is poor, the outcomes of the computer simulation will have partial validity and it is likely that the intended users will have limited confidence in the results of the study. In fact, a large number of healthcare M\&S 
studies fail because stakeholders have not been considered during the development phases of the model (Brailsford et al, 2009a, b; Jahangirian et al, 2015). We observe a lack of deployment of wider soft OR methods in simulation requirement capture leading potentially to simulation designs which are sub-optimal with respect to the inclusion of the widest possible sources of system knowledge and benefit valuations of the system outputs. While soft system methods are seen as uniquely powerful in eliciting multiple viewpoints from stakeholders and system owners, we encourage the deployment of those system representation methods such as QSD which can accommodate multiple viewpoints but in such a way as to communicate directly with the simulation requirements process.

Work is in hand to establish the practicality of using the toolset of QSD (Powell and Coyle, 2005) not only in the area of health system representation and assessment, but in the associated fields of (safety and mission) critical human activity systems (CHASs) such as disaster and crisis management (Powell et al, 2016) and high-risk environments. This work illustrates the need for a complementary approach, in which it illustrates the diverse ontologies of components of the system-in-focus. Specifically, a typical CHAS contains three intersecting systems, namely: the physical (having a positivist, singly defined and accessible reality); the policy context (being socially constructed, but singly defined through the exertion of power and appearing in its effect on the physical reality as a measurable set of effects); and the social system, which includes the experiences and individually, socially constructed valuations of the system inhabitants.

Our observations on the literature have led us to consider the relationship between these various components of a policy assessment regime. We observe a complementarity between broad policy assessment methods which have to take into account multiple, often ill-defined views and more focused and precise system representations through the use of simulations, a complementarity which is, perhaps necessarily, an uneasy one. The simulation seeks precision in requirement, and the broad policy method seeks engagement, breadth of representation and generality, but in order to support the process of sound policy identification and hence assessment, these two must work together.

We observe a lack of deployment of wider soft OR methods in simulation requirement capture leading potentially to simulation designs which are sub-optimal with respect to the inclusion of the widest possible sources of system knowledge and benefit valuations of the system outputs. While soft system methods are seen as uniquely powerful in eliciting multiple viewpoints from stakeholders and system owners, we encourage the deployment of those system representation methods such as QSD which can accommodate multiple viewpoints but in such a way as to communicate directly with the simulation requirements process.
Acknowledgements-This paper is based on a keynote lecture at OR56, 2014. We wish to thank various colleagues at that conference, the reviewers and the editors for the valuable comments and suggestions.

\section{References}

Barros FJ (1995). Dynamic structure discrete event system specification: A new formalism for dynamic structure modeling and simulation. In Proceedings of the 27th Conference on Winter Simulation Conference, Arlington, VA, pp. 781-785.

Bérard C (2010). Group model building using system dynamics: An analysis of methodological frameworks. Electronic Journal of Business Research Methods 8(1):35-46.

Brailsford SC, Bolt T, Connell C, Klein JH and Patel B (2009). Stakeholder engagement in health care simulation. In: Proceedings of the 2009 Winter Simulation Conference, Austin, TX, pp. 1840-1849.

Brailsford SC, Harper PR, Patel B and Pitt M (2009). An analysis of the academic literature on simulation and modelling in healthcare. Journal of Simulation 3(3):130-140.

Brailsford SC, Desai SM and Viana J (2010). Towards the Holy Grail: Combining system dynamics and discrete-event simulation in healthcare. In: Proceedings of the 2010 Winter Simulation Conference, Baltimore, MA, pp. 2293-2303.

Chahal K (2010). A Generic Framework for Hybrid Simulation in Healthcare. PhD Thesis. Brunel University, UK.

Chahal K and Eldabi T (2008). Applicability of hybrid simulation to different modes of governance in UK healthcare. In: Proceeding of the 2008 Winter Simulation Conference, Piscataway, New Jersey, pp. 1469-1476.

Chahal K and Eldabi T (2010). A generic framework for hybrid simulation in healthcare. In: Proceedings of the 28th International Conference of the System Dynamics Society, Seoul, Korea, pp. 526-541.

Checkland, P. (1981). Systems thinking systems practice. Wiley: Chichester, UK.

Checkland P and Scholes J (1990). Soft Systems Methodology in Action. Wiley: Chichester, UK.

Churchman CW (1967). Wicked problems-Guest editorial. Management Science 14(4):B141-B142.

Daellenbach HG (n.d.). Hard OR, Soft OR, problem structuring methods, critical systems thinking: A primer. Retrieved from http:// orsnz.org.nz/conf36/papers/Daellenbach.pdf. Retrieved 24 May 2016.

Demirkan H and Delen D (2013). Leveraging the capabilities of service-oriented decision support systems: Putting analytics and big data in cloud. Decision Support Systems 55(1):412-421.

Djanatliev A and German R (2013). Prospective healthcare decisionmaking by combined system dynamics, discrete-event and agentbased simulation. In: Proceedings of the 2013 Winter Simulation Conference, Washington, DC, pp. 270-281.

Djanatliev A, German R, Kolominsky-Rabas P and Hofmann BM (2012). Hybrid simulation with loosely coupled system dynamics and agent-based models for prospective health technology assessments. In: Proceedings of the 2012 Winter Simulation Conference, Berlin, pp. 770-781.

Eden C (1989). Using cognitive mapping for strategic options development and analysis (SODA). In: Rosenhead J (ed) Rational Analysis for a Problematic World. Wiley: Chichester, UK.

Eden C and Ackermann F (2004). Cognitive mapping expert views for policy analysis in the public sector. European Journal of Operational Research 152(3):615-630.

Eldabi T, Jun T, Clarkson J, Connell C and Klein J (2010). Modeldriven health care: Disconnected practices. In: Proceedings of the 
2010 Winter Simulation Conference, Baltimore, Maryland, pp. 2271-2282.

EPSRC/ESRC (2004). Review of research status of operational research in the UK. https://www.epsrc.ac.uk/newsevents/pubs/ review-of-research-status-of-operational-research-in-the-uk/. Accessed 25 May 2016.

Fakhimi M, Mustafee N and Stergioulas L (2015). An investigation of hybrid simulation for modeling sustainability in healthcare. In: Proceedings of the 2015 Winter Simulation Conference, Huntington Beach, CA, pp. 1585-1596.

Hollocks BW (2006). Forty years of discrete-event simulation-A personal reflection. Journal of the Operational Research Society 57(12):1383-1399.

Jackson MC (2000). System Approaches to Management. Springer: New York, NY.

Jahangirian M, Taylor SJE, Eatock J, Stergioulas LK and Taylor PM (2015). Causal study of low stakeholder engagement in healthcare simulation projects. Journal of the Operational Research Society 66(3):369-379.

Katsaliaki K and Mustafee N (2011). Applications of simulation research within the healthcare context. Journal of the Operational Research Society 62(8):1431-1451.

Kotiadis K, Tako A and Vasilakis C (2014). A participative and facilitative conceptual modelling framework for discrete event simulation studies in healthcare. Journal of the Operational Research Society 65(2):197-213.

Kotiadis K (2007). Using soft system methodology to determine the simulation study objectives. Journal of Simulation 1(3): 215-222.

Law AM (1991). Simulation model's level of detail determines effectiveness. Industrial Engineering 23(10):16-18.

Lee T, Cho S-H, Jang H and Turner JG (2012). A simulation-based iterative method for a trauma center-Air ambulance location problem. In: Proceedings of the 2012 Winter Simulation Conference, Berlin, pp. 955-966.

Lehaney B and Paul RJ (1996). The use of soft systems methodology in the development of a simulation of out-patient services at Watford General Hospital. Journal of the Operational Research Society 47(4):864-870.

Lehaney B and Paul RJ (1994). Using soft systems methodology to develop a simulation of outpatient services. Journal of the Royal Society for the Promotion of Health 114(5):248-251.

Lendermann P, Gan BP and McGinnis LF (2001). Distributed simulation with incorporated APS procedures for high-fidelity supply chain optimization. In: Proceedings of the 33rd Winter Simulation Conference, Arlington, VA, pp 1138-1145.

Liddell W and Powell JH (2004). Agreeing access policy in a general medical practice: A case study using QPID. System Dynamics Review 20(1):49-73.

Maria A (1997). Introduction to modeling and simulation. In Proceedings of the 29th Conference on Winter Simulation Conference, Atlanta, GA, pp. 7-13.

Mingers J and Lyons L (2009). A review of the recent contribution of system thinking to operational research and management science. Working Paper series no 197, University of Kent Business School, Kent, UK. ISSN 1741-7597.

Mustafee N, Taylor SJE, Katsaliaki K and Brailsford SC (2009). Facilitating the analysis of a UK national blood service supply chain using distributed simulation. Simulation: Transactions of the Society of Modelling and Simulation International 85(2):113-128.

Mustafee N and Taylor SJE (2009). Speeding up simulation applications using WinGrid. Concurrency and Computation: Practice and Experience 21(11):1504-1523.

Mustafee N, Katsaliaki K, Bischoff EE and Williams MD (2012). Proximity-based modelling of cross-contamination through agentbased simulation: a feasibility study. Health Systems 2(1):61-71.
Mustafee N, Sahnoun M, Smart A, Godsiff P, Baudry D and Louis A. (2015). Investigating execution strategies for hybrid models developed using multiple M\&S methodologies. In: Proceedings of the 2015 Spring Simulation Multi-Conference (SpringSim'15)ANSS symposia, April 12-15, 2015, Alexandria, VA.

Park H and Fishwick PA (2010). A gpu-based application framework supporting fast discrete-event simulation. Simulation, 86(10): 613-628.

Perumalla KS (2006). Discrete-event execution alternatives on general purpose graphical processing units (GPGPUs). In: Proceedings of the 20th Workshop on Principles of Advanced and Distributed Simulation. Singapore, pp. 74-81.

Powell JH and Coyle RG (2005). Identifying strategic action in highly politicized contexts using agent-based qualitative SD. Journal of the Operational Research Society 56(7):787-798.

Powell JH, Mustafee N, Chen AS and Hammond M (2016). Systemfocused risk identification and assessment for disaster preparedness: Dynamic threat analysis. European Journal of Operational Research 254(2):550-564.

Powell JH and Swart J (2010). Mapping the values in B2B relationships. Industrial Marketing Management 39(3):437-439.

Robinson S (2008) Conceptual modelling for simulation. Part 1. Definition and requirements. Journal of the Operational Research Society 59(3):278-290.

Stubbs L, Howard N and Tait A (n.d.). How to model a confrontation-Computer support for drama theory. Retrieved from http:// www.decisionmechanics.com/wp-content/uploads/2009/11/ ccrts 1999.pdf. Accessed 27 May 2016.

Swart J and Powell JH (2006). Men and measures: Capturing knowledge requirements in firms through qualitative system modeling. Journal of the Operational Research Society 57(1):10-21.

Robinson S (2011). Choosing the right model: Conceptual modeling for simulation. In: Proceedings of the 2011 Winter Simulation Conference, Phoenix, Arizona, pp. 1423-1435.

Sterman, J.D. (2000). Business Dynamics: Systems Thinking and Modeling for a Complex World. McGraw-Hill/Irwin: Boston, MA.

Traoré MK (2003). Foundations of multi-paradigm modeling and simulation: A meta-theoretic approach to modeling and simulation. In: Proceedings of the 2003 Winter Simulation Conference, New Orleans, LA, pp. 604-612.

Taylor SJE, Ghorbani M, Mustafee N, Turner SJ, Kiss T, et al (2011). Distributed computing and modeling \& simulation: Speeding up simulations and creating large models. In: Proceedings of the 2011 Winter Simulation Conference, Phoenix, AZ, pp. 161-175.

Ulrich W (1988). Systems thinking systems practice and practical philosophy: A program of research. Systems Practice 1(2): 137-163.

Viana J, Brailsford SC, Harindra V and Harper PR (2014). Combining discrete-event simulation and system dynamics in a healthcare setting: A composite model for Chlamydia infection. European Journal of Operational Research 237(1):196-206.

Viana J, Rossiter S, Channon AA, Brailsford SC and Lotery A (2012). A multi-paradigm, whole system view of health and social care for age-related macular degeneration. In: Proceedings of the 2012 Winter Simulation Conference, Berlin, pp 1070-1081.

Vidal RVV (2005). Soft OR approaches. Engevista 7(1):4-20.

Zulkepli J, Eldabi T and Mustafee N (2012). Hybrid simulation for modelling large systems: An example of integrated care model. In: Proceedings of the 2012 Winter Simulation Conference, Berlin, pp. 758-769.

Received 17 October 2014; accepted 8 November 2016 